\title{
Statistical Properties of the Immediate Aftershocks of the 15 October 2013 Magnitude 7.1 Earthquake in Bohol, Philippines
}

\author{
Rene C. BATAC \\ National Institute of Physics, University of the Philippines, \\ Quezon City, Philippines; e-mail: rbatac@nip.upd.edu.ph
}

\begin{abstract}
The aftershock records of the magnitude 7.1 earthquake that hit the island of Bohol in central Philippines on 15 October 2013 is investigated in the light of previous results for the Philippines using historical earthquakes. Statistics of interevent distances and interevent times between successive aftershocks recorded for the whole month of October 2013 show marked differences from those of historical earthquakes from two Philippine catalogues of varying periods and completeness levels. In particular, the distributions closely follow only the regimes of the historical distributions that were previously attributed to the strong spatio-temporal correlations. The results therefore suggest that these correlated regimes which emerged naturally from the analyses are strongly dominated by the clustering of aftershock events.
\end{abstract}

Key words: earthquakes, interevent distances, interevent times, time series analysis.

\section{INTRODUCTION}

The magnitude $\left(M_{w}\right) 7.1$ earthquake that affected the island of Bohol in central Philippines on 15 October 2013 resulted in the death of 222 people and economic losses amounting to 52.06 US dollars due to the destruction of infrastructure (Lagmay and Eco 2014), including cultural heritage buildings

Ownership: Institute of Geophysics, Polish Academy of Sciences;

(c) 2015 Batac. This is an open access article distributed under the Creative Commons

Attribution-NonCommercial-NoDerivs license,

http://creativecommons.org/licenses/by-nc-nd/3.0/. 
like the Spanish-era churches of Baclayon and Loboc, believed to be some of the oldest in the country (Revilla 2014). Apart from the economic and death toll, however, the quake is of particular importance as it revealed a previously unknown fault or fault system in the northern part of the island (Lagmay and Eco 2014, Kobayashi 2014). Analysis of the earthquake and its immediate aftershocks can therefore reveal much about this newly discovered seismogenic zone.

To this end, a useful first approach would be to investigate the spatiotemporal properties of the generated earthquakes. Analyses based on interevent distances (or epicentre separation distances) and interevent times (or recurrence/waiting times) between successive events have been studied using global or regional earthquake records, revealing similarities and differences between earthquake-generating mechanisms (Batac and Kantz 2014, Marekova 2014). In particular, Batac and Kantz (2014) observed that both interevent distance and interevent time distributions exhibit two characteristic regimes corresponding to the clustering of correlated earthquakes at short spatio-temporal scales and the corresponding separation of independent events at longer separation distances and waiting times. The work includes the statistical distributions obtained for historical Philippine earthquakes (1973-2012) obtained from the global Preliminary Determination of Epicentres Catalogue (PDE 1973-2012).

In this work, the spatio-temporal behavior of the immediate aftershocks of the magnitude 7.1 Bohol earthquake (15-21 October 2013) is characterized in the light of the previous results by Batac and Kantz (2014) using historical data. Interestingly, the observed spatio-temporal distributions closely follow those of the historical earthquakes only for short interevent distances and interevent times, and lack the secondary peaks observed at longer space and time scales. This suggests that the short interevent distance and interevent time regimes are primarily dominated by the aftershock occurrences.

\section{EARTHQUAKE DATA SETS AND ANALYSIS}

The work utilizes two earthquake records for the Philippine region, with different periods and sensitivities. Historical earthquakes from 1973-2012 used by Batac and Kantz (2014), obtained from the PDE Catalogue (1973-2012) at the region $4-24^{\circ} \mathrm{N}$ and $115-130^{\circ} \mathrm{E}$ containing the Philippine archipelago, is used for comparison with events generated by 15 October Bohol earthquake. This record contains $10000+$ events that are above the threshold magnitude $M_{t h}=4.5$ that ensures substantial completeness.

The second historical record is obtained from the Philippine Institute of Seismology and Volcanology (PHIVOLCS) for the period 2011-2013 (PHIVOLCS 2013). The PHIVOLCS Catalogue has a record of 4000+ earth- 

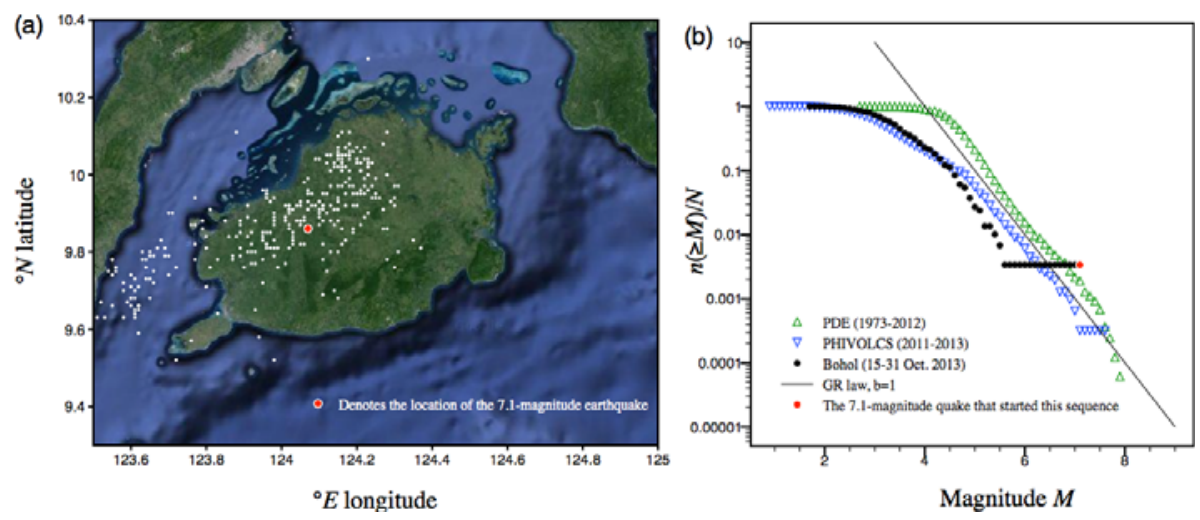

Fig. 1: (a) Spatial distribution of Bohol earthquake aftershocks for the whole month of October 2013; (b) Cumulative magnitude count $n(\geq M)$ divided by the total number of events $N$ for the different data sets considered. Both PDE and PHIVOLCS earthquake data begin to obey the Gutenberg-Richter law at around $\mathrm{M}_{t h}=4.5$. Within the month of October 2013, the 7.1-magnitude Bohol earthquake produced relatively strong aftershocks starting with $M=5.5$, but subsequent records appear to follow a distribution similar to the PHIVOLCS record.

quakes within the specified period and $M_{t h}$. Of these events, 234 happened within the 15-31 October 2014 time window and classified as aftershocks of the 7.1-magnitude Bohol earthquake. Figure 1 shows the spatial distribution of epicentres and the normalized cumulative magnitude distribution of the PDE, PHIVOLCS, and Bohol data sets.

For the earthquake records used, the space and time separation measures between successive earthquakes in the sequence are calculated. Upon considering $N$ earthquake events in the catalogue, the $i^{\text {th }}$ event at $\left(\phi_{i}, \theta_{i}\right)$ (where $\phi$ and $\theta$ denote the epicentre longitude and latitude coordinates, respectively) will be separated from the $(i+1)$ th event at $\left(\phi_{i+1}, \theta_{i+1}\right)$ by the interevent distance $R_{i}$

$$
R_{i}=R_{E} \arccos \left(\sin \phi_{i} \sin \phi_{i+1}+\cos \phi_{i} \cos \phi_{i+1}+\cos \left|\theta_{i}-\theta_{i+1}\right|\right),
$$

where $R_{E}=6371 \mathrm{~km}$ is the approximate radius of the Earth. Correspondingly, the same two events will have occurrence times $t_{i}$ and $t_{i+1}$, and will therefore be separated in time by the interevent time $T_{i}$

$$
T_{i}=t_{i+1}-t_{i} .
$$

The set of all $R_{i}$ 's and $T_{i}$ 's are collectively denoted by $R$ and $T$, respectively, and analyzed based on their distribution functions. The relative frequency distributions $f(R)$ and $f(T)$ denote the actual counts of a particular 
value of $R$ and $T, n(R)$ and $n(T)$, divided by the total number in the set, which is just equal to $N-1$ (i.e., because the last event considered has no succeeding event). Touati et al. (2009) showed that several features of the distributions, which are not readily discernible upon normalization, could be captured upon presenting relative frequency distributions. On the other hand, the probability density functions ( $\mathrm{pdfs}) p(R)$ and $p(T)$ report the distributions normalized with both $N-1$ and the bin widths used to present the data.

There is a wealth of previous literature that attempt to obtain the approximate form of the $p(R)$ (Davidsen and Paczuski 2005, Corral 2006, Batac and Kantz 2014, Marekova 2014) and $p(T)$ (Bak et al. 2002, Corral 2004, Davidsen and Goltz 2004, Touati et al. 2009, Batac and Kantz 2014) based on empirical distributions obtained from historical records of different seismogenic regions. Inspired by the universality of power-law Gutenberg-Richter distributions of earthquake magnitudes (Gutenberg and Richter 1954), Bak et al. (2002) attributed the scaling behavior of earthquakes to self-organized criticality, a dynamical state wherein the system reaches a critical state without a fine-tuning of parameters. Succeeding authors also noted power-law regimes in the distributions of interevent distances for regional (Davidsen and Paczuski 2005, Marekova 2014) and global (Corral 2006) records. Interevent times are also fitted with distributions containing power-law sections (Corral 2004, Davidsen and Goltz 2004). In particular, Corral (2004) observed that the distributions collapse under proper scaling under a curve that is best described by a gamma distribution. Succeeding work by Lippiello et al. (2012) and Marekova (2012) also noted the regimes of the distributions where the data deviates from the gamma distribution. On the other hand, other succeeding studies suggest that these distributions are substantially non-universal and resulting from a crossover between two regimes of different characteristic time scales (Touati et al 2009, Batac and Kantz 2014).

Therefore, in this work, the aim is not to determine the best fitting function for the resulting distributions, but to establish characteristic separation distance and time scales involved in regional seismicity. Our analyses benefit from the availability of both historical data and "fresh" aftershock sequences, providing a good comparison between all earthquakes in the catalogue and earthquakes generated by the same main shock. In the succeeding, we show that the aftershock sequences follow the historical distributions only for the regimes previously determined to be dominated by correlated earthquakes (Batac and Kantz 2014).

\section{DISTRIBUTIONS OF INTEREVENT DISTANCES AND TIMES}

It has been established that the distribution of separation distances between earthquakes from regional catalogues follows similar distributions across a 

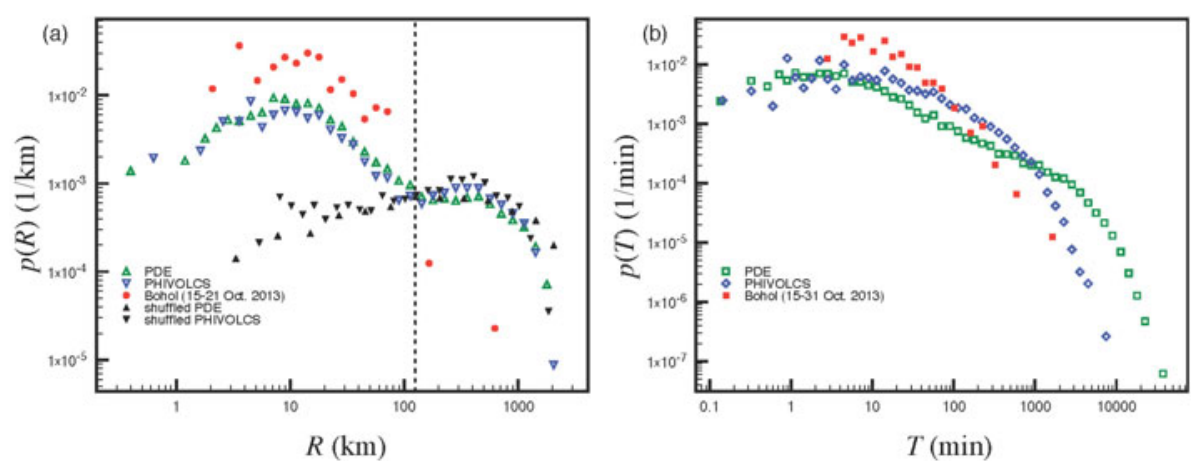

Fig. 2. Probability density functions of: (a) interevent distances, $p(R)$, and (b) interevent times, $p(T)$. In panel (a) the $p(R)$ shows two regimes for historical PDE and PHIVOLCS, while the Bohol aftershock sequence is unimodal, with a trend closely following only the short- $R$ regimes. In contrast, the shuffled historical data is shown to mimic the long- $R$ regime, suggesting that this regime is dominated by events that have no correlation. The characteristic separation distance $R^{*} \approx$ $125 \mathrm{~km}$ where the original and shuffled sequences begin to show comparable trends is denoted by the broken line. In panel (b) the $p(T)$ distributions show different cutoff regimes, which is expected due to the different lengths of time periods of the various catalogs.

range of magnitudes that ensure data completeness (Batac and Kantz 2014, Marekova 2014). The regimes of longer $R$ values, in particular, are limited to the chosen observation window, resulting in a finite-size tapering off toward the diagonal of the rectangular window considered. On the other hand, short$R$ regimes are expected to be dominated by the clustering behavior of related earthquakes.

In Fig. 2a, the probability density functions of $R, p(R)$, for the historical earthquakes from the PDE and PHIVOLCS catalogues are presented along with the corresponding distributions from the Bohol aftershock sequence. Qualitatively, the distributions show a separation between two regimes of different characteristic $R$ scales, which is believed to be a manifestation of two spatial regimes involved in seismicity. The short- $R$ regime may be attributed to the spatial clustering of correlated events, like aftershocks or earthquake swarms; on the other hand, the long- $R$ part of the distributions results from the spatial separation between independent events.

The origin of the long- $R$ regime may be probed by comparing the actual distributions with randomly shuffled sequences. Batac and Kantz (2014) conducted shuffling in the temporal domain by dividing the entire time of observation into small time slices and shuffling all these time slices. Time slices that contain earthquake events therefore retain the magnitude and epi- 
centre location information and only receive a new occurrence time upon shuffling. Such procedure removes any form of correlation among successive events in the original time sequence. The same procedure is employed in the analyses, and the resulting pdf's are shown in Fig. 2a. Consistent with previous results, the pdf's of shuffled events for both PDE and PHIVOLCS closely replicate the long- $R$ regime, establishing the uncorrelated nature of events that produce this part of the distribution.

What about the short- $R$ regime? Previous works propose that, in contrast with the long- $R$ regime, the short- $R$ regimes originate from correlated earthquakes. This explanation appears to be validated by the resulting statistics of Bohol aftershocks. In contrast with the historical data, the distribution from the Bohol sequence is clearly unimodal with peak at around $10 \mathrm{~km}$, as shown in Fig. 2a. More importantly, the distribution of separation distances between Bohol aftershocks is found to closely replicate the trend of the short- $R$ regime from the historical data. While we cannot remove the possible contributions from other form of correlated earthquake events (e.g., earthquake swarms), the data presented in Fig. 2 clearly shows that aftershocks follow the short- $R$ regime, establishing one possible origin of these parts of the historical distribution.

The corresponding interevent time distributions $p(T)$ are presented in Fig. 2b. Because of the differences in the periods considered, the probability of occurrence of longer waiting times is significantly higher for the PDE record that contains earthquake sequences taken from 1973-2012. Even so, it can be observed that the PHIVOLCS record, containing earthquakes from 2011-2013, shows a behavior comparable to the PDE. In Fig. 2b, this regime is observed for intermediate $T$ values (around 10-1000 min); incidentally, this regime is usually fitted by power-law curves due to the almost linear trend in a double logarithmic scale. Finally, the Bohol record, apart from being very short, does not appear to have any similarities with the historical distributions. In the following section, conditional distributions are used to make sense of the resulting Bohol interevent time statistics.

\section{SPATIO-TEMPORAL CONDITIONAL DISTRIBUTIONS}

Because aftershock sequences exhibit high clustering both in space and time scales, conditional distributions of interevent times subject to the corresponding interevent distance will reveal the underlying spatio-temporal correlation. As a first approximation, the intersection between the interevent distance distributions of original and shuffled sequences may be used to denote "nearby" and "away" events.

In Fig. 2a, the intersection of the original and shuffled historical sequences is marked by a broken line dividing the $p(R)$ distribution. This characteristic $R^{*}$, which is found to be approximately constant for different 

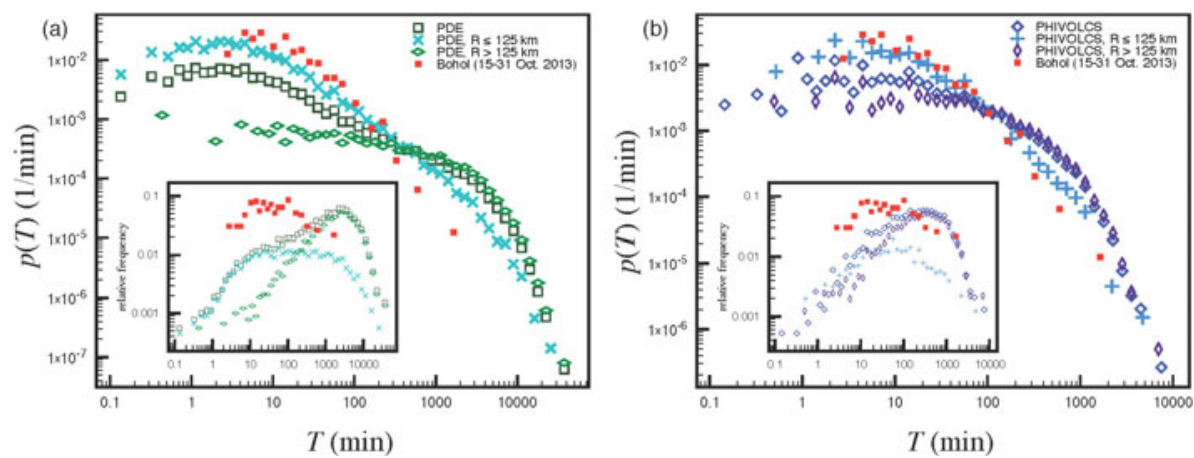

Fig. 3. Conditional distributions of $T$ subject to the corresponding $R$ relative to the characteristic value $R^{*} \approx 125 \mathrm{~km}$ obtained from Fig. 2a. The PDE (a) and PHIVOLCS (b) data are presented as probability density plots, with relative frequency plots in the insets. The $p(T)$ and the conditional distributions $p\left(T \mid R \leq R^{*}\right)$ and $f\left(T \mid R>R^{*}\right)$ do not collapse under the same curve; in the inset, this is observed as a result of the difference between $f\left(T \mid R \leq R^{*}\right)$ and $f\left(T \mid R>R^{*}\right)$, with the total $f(R)$ emerging as a crossover between the two conditional distributions. The distributions obtained from the Bohol aftershock sequences are comparable to the behavior of the short-distance component of the total distribution.

threshold magnitudes and previously measured to be around $125 \mathrm{~km}$ for the Philippines (Batac and Kantz 2014), is then used to obtain conditional relative frequency distributions $f\left(T \mid R \leq R^{*}\right)$ and $f\left(T \mid R>R^{*}\right)$, and the corresponding conditional probability density functions $p\left(T \mid R \leq R^{*}\right)$ and $p\left(T \mid R>R^{*}\right)$. These distribution functions, along with those of the Bohol sequence, are presented in Fig. 3.

The plots show differences in the behavior of the distributions that persist even upon normalization. The normalized distributions $p(T), p\left(T \mid R \leq R^{*}\right)$ and $p\left(T \mid R>R^{*}\right)$, as shown in Fig. 3, do not collapse under the same curve upon normalization. Instead, for short $T$ values, $p\left(T \mid R>R^{*}\right)<p(T)<$ $p\left(T \mid R \leq R^{*}\right)$, suggesting "spatio-temporal clustering", i.e., events separated by shorter distances are more likely to be separated by shorter waiting times; on the other hand, for very long $T$ values near the tails of the distributions, $p\left(T \mid R \leq R^{*}\right)<p(T)<p\left(T \mid R>R^{*}\right)$, showing "spatio-temporal separation", i.e., events happening at longer separation distances, which are more likely to be uncorrelated, also happen at longer characteristic waiting times.

It is also notable that the obtained conditional relative frequency distributions support the previous works showing that the interevent time distributions result from the crossover between two distributions of different characteristic times (Touati et al. 2009). In the insets of Fig. 3, this crossover is clearly highlighted upon plotting the distribution of all interevent times, 
$f(T)$, with $f\left(T \mid R \leq R^{*}\right)$ and $f\left(T \mid R>R^{*}\right)$. The $f\left(T \mid R \leq R^{*}\right)$ distributions peak at values around 10-100 min, while the $f\left(T \mid R>R^{*}\right)$ show peaks at $200 \mathrm{~min}$ for the PHIVOLCS and at $1000 \mathrm{~min}$ for the PDE data. The similarities in the peak values of $f\left(T \mid R \leq R^{*}\right)$ is hinted to be resulting from common mechanisms governing correlated earthquake sequences, while the differences in the $f\left(T \mid R>R^{*}\right)$ is attributed to the different time scales involved in independent events which is also related to the length of the catalogue (Batac and Kantz 2014). Interestingly, the unimodal behavior and the peak value of the $f\left(T \mid R \leq R^{*}\right)$ distributions is comparable to that of the Bohol aftershock sequence. This result highlights the fact that the correlated mechanisms generating the short $R$ and $T$ for correlated earthquakes result primarily from aftershock sequences, earthquake generated by the same main shock.

For better comparison, Fig. 4 shows the conditional probability density functions $p\left(T \mid R \leq R^{*}\right)$ and $p\left(T \mid R>R^{*}\right)$ separately along with the Bohol aftershocks $p(T)$. In Fig. 4a, the Bohol waiting time pdfs show remarkable comparison with historical $p\left(T \mid R \leq R^{*}\right)$, especially at the short $T$ values. Because of the very short period considered (15-31 October 2013) for the aftershock distributions, the tail of the Bohol aftershock $T$ distributions taper off faster than those of the historical $p\left(T \mid R \leq R^{*}\right)$. In contrast, the regimes of long $R$ and long $T$ have different characteristic values resulting in stark differences between the $p\left(T \mid R>R^{*}\right)$ and the Bohol $p(T)$ distributions in Fig. 4b.
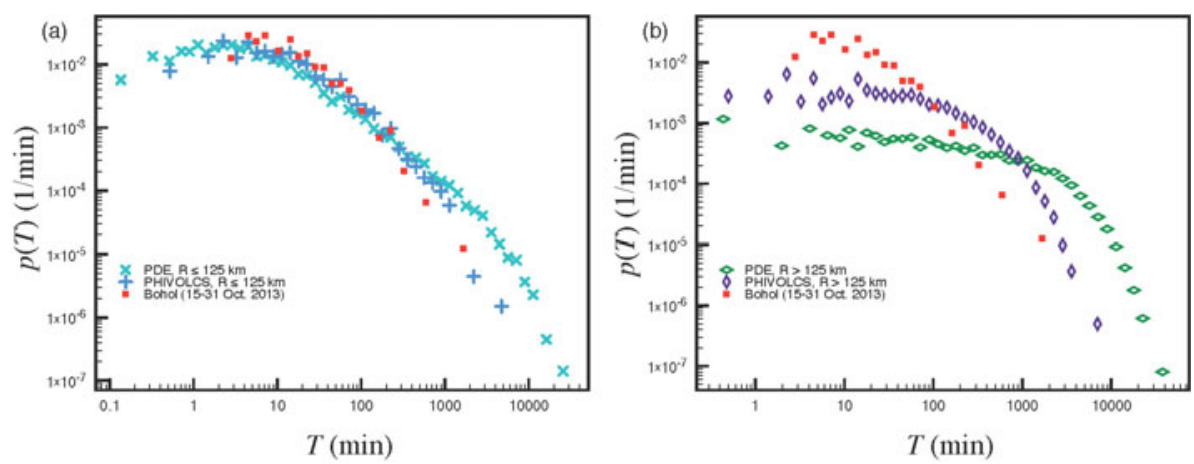

Fig. 4. The same $p\left(T \mid R \leq R^{*}\right)$ (panel (a)) and $p\left(T \mid R>R^{*}\right.$ ) (panel (b)) distributions from Fig. $3 \mathrm{~b}$ and d, plotted separately, and with the Bohol aftershocks $p(T)$. In panel (a) the short- $R$ and short- $T$ plots have comparable statistical distributions, suggesting universal generation mechanisms. Because the Bohol aftershock $p(T)$ appears to follow the same behavior, we can infer that these conditional distributions are primarily due to the spatio-temporal clustering of aftershock sequences. In panel (b), on the other hand, the long- $R$ and long- $T$ plots generated by independent events show no such similarities. 
The similarities of the short- $R$ conditional $T$ distributions among themselves and with the Bohol aftershock pdf suggests that this regime, dominated by successive events separated by short distances and equally short waiting times, is primarily due to aftershock sequences. The 7.1-magnitude earthquake in Bohol, which provided clear identification of aftershock locations and arrival times, therefore supports previous results that points to correlated mechanisms as the origin of this component of the total $T$ distributions (Batac and Kantz 2014).

\section{CONCLUSION}

The immediate aftershocks of the 7.1-magnitude Bohol earthquake taken within the period of 15-31 October 2013 offered fresh insights into the problem of spatio-temporal characterization of earthquake occurrences. The statistics of interevent times and interevent distances between successive earthquake events in the Bohol series clearly lack the long-distance and long-time components observable in historical data. On the other hand, the statistics of the Bohol aftershock sequence shows remarkable similarities with distributions of conditional distributions obtained from short-distance and short-time events. This suggests that this component, which is also obtained from historical data (Batac and Kantz 2014) and from numerical models (Touati et al. 2009), is dominated by the occurrence of correlated aftershocks.

The results agree with very recent analyses of model and empirical data (Touati et al. 2009, Marekova 2014) and also point to the efficacy of the techniques presented in a previous work for separating the correlated and uncorrelated components from the total distributions of interevent distances and interevent times (Batac and Kantz 2014). The technique does so without imposing arbitrary space and time scales: for the interevent distance, the boundary separating the short and long distance events is obtained by simple comparison with a randomly shuffled sequence, while for the interevent times, the conditional distributions subject to the corresponding interevent times are used to generate the component distributions. For the case of the Philippine seismogenic region, the comparison with the Bohol sequence clearly showed that the characteristic values emergent from the analyses are not arbitrary, as they are comparable to the statistical distributions obtained from "fresh" aftershock sequences. The technique may therefore be extended for other seismogenic regions and even for finer observation scales. Finally, the origin and implications of the observed clustering behavior may be understood better by incorporating insights from associated numerical models of seismicity and related phenomena (Touati et al. 2009, Batac et al. 2012, Białecki and Czechowski 2013). 
Acknowledgments. The work is supported by grants from the National Institute of Physics (NIP) and the University of the Philippines Diliman Office of the Vice Chancellor for Research and Development (OVCRD).

\section{References}

Bak, P., K. Christensen, L. Danon, and T. Scanlon (2002), Unified scaling law for earthquakes, Phys. Rev. Lett. 88, 17, 178501, DOI: 10.1103/PhysRevLett. 88.178501 .

Batac, R.C., and H. Kantz (2014), Observing spatio-temporal clustering and separation using interevent distributions of regional earthquakes, Nonlin. Processes Geophys. 21, 4, 735-744, DOI: 10.5194/npg-21-735-2014.

Batac, R., A. Longjas, and C. Monterola (2012), Statistical distributions of avalanche size and waiting times in an inter-sandpile cascade model, Physica A 391, 3, 616-624, DOI: 10.1016/j.physa.2011.08.032.

Białecki, M., and Z. Czechowski (2013), On one-to-one dependence of rebound parameters on statistics of clusters: Exponential and inverse-power distributions out of Random Domino Automaton, J. Phys. Soc. Jpn. 82, 1, 014003, DOI: $10.7566 / J P S J .82 .014003$.

Corral, A. (2004), Long-term clustering, scaling, and universality in the temporal occurrence of earthquakes, Phys. Rev. Lett. 92, 10, 108501, DOI: 10.1103/ PhysRevLett.92.108501.

Corral, A. (2006), Universal earthquake-occurrence jumps, correlations with time, and anomalous diffusion, Phys. Rev. Lett. 97, 17, 178501, DOI: 10.1103/ PhysRevLett.97.178501.

Davidsen, J., and C. Goltz (2004), Are seismic waiting time distributions universal? Geophys. Res. Lett. 31, 21, L21612, DOI: 10.1029/2004GL020892.

Davidsen, J., and M. Paczuski (2005), Analysis of the spatial distribution between successive earthquakes, Phys. Rev. Lett. 94, 4, 048501, DOI: 10.1103/ PhysRevLett.94.048501.

Gutenberg, B., and C.F. Richter (1954), Seismicity of the Earth and Associated Phenomena, 2nd ed., Princeton University Press, Princeton.

Kobayashi, T. (2014), Remarkable ground uplift and reverse fault ruptures for the 2013 Bohol earthquake (Mw 7.1), Philippines, revealed by SAR pixel offset analysis, Geosci. Lett. 1, 1, 7, DOI: 10.1186/2196-4092-1-7.

Lagmay, A.M.F., and R. Eco (2014), The magnitude 7.2 Bohol earthquake, Philippines, Nat. Hazards Earth Syst. Sci. Discuss. 2, 2103-2115, DOI: 10.5194/ nhessd-2-2103-2014. 
Lippiello, E., Á. Corral, M. Bottiglieri, C. Godano, and L. de Arcangelis (2012), Scaling behavior of the earthquake intertime distribution: Influence of large shocks and time scales in the Omori law, Phys. Rev. E 86, 6, 066119, DOI: 10.1103/PhysRevE.86.066119.

Marekova, E. (2012), Testing a scaling law for the earthquake recurrence time distributions, Acta Geophys. 60, 3, 858-873, DOI: 10.2478/s11600-012$0007-\mathrm{y}$.

Marekova, E. (2014), Analysis of the spatial distribution between successive earthquakes occurred in various regions in the world, Acta Geophys. 62, 6, 1262-1282, DOI: 10.2478/s11600-014-0234-5.

PDE (1973-2012), Preliminary Determination of Epicentres Catalogue, http:// earthquake.usgs.gov/earthquakes/eqarchives/epic, last access: December 2012.

PHIVOLCS (2013), Philippine Institute of Seismology and Volcanology Catalogue, http://www.phivolcs.dost.gov.ph/html/update_SOEPD/EQLatest.html, last access: May 2014.

Revilla, L.S. (2014), Protecting our cultural heritage: A Herculean task. In: $A C C U$ Nara International Correspondent, Vol. 13, Cultural Heritage Protection Cooperation Office, Asia-Pacific Cultural Centre for UNESCO (ACCU), Nara, Japan, 44-47.

Touati, S., M. Naylor, and I.G. Main (2009), Origin and nonuniversality of the earthquake interevent time distribution, Phys. Rev. Lett. 102, 16, 168501, DOI: 10.1103/PhysRevLett.102.168501. 